(2) Open Access Full Text Article

\title{
Lessons learned from introducing huddle boards to involve nursing staff in targeted observation and reporting of medication effect in a nursing home
}

This article was published in the following Dove Press journal:

Journal of Multidisciplinary Healthcare

\author{
Stephan Ore' \\ Elin Olaug Rosvold ${ }^{2}$ \\ Ragnhild Helles $\varnothing^{3}$ \\ 'Oppsalhjemmet Nursing Home \\ Norlandia, NO-0982 Oslo, Norway; \\ 2Department of General Practice, \\ Institute of Health and Society, \\ Faculty of Medicine, University \\ of Oslo, NO-0318 Oslo, Norway; \\ ${ }^{3}$ Department of Nursing Sciences, \\ Institute of Health and Society, \\ Faculty of Medicine, University of \\ Oslo, NO-03।8 Oslo, Norway
}

\begin{abstract}
Background: Medication administration and management in nursing homes can occur during all phases of the medication process. The aim of this study was to investigate if an introduction of a systematic use of huddle board led to an increased amount of documentation in the patient record of observations of effects and side effects following a change in medication.
\end{abstract}

Methods: A three-layer intervention approach combining huddle boards, educating the entire staff in medication observation and documentation, and frequent feedback to the staff about the outcome was applied. A standard was set for the expected reporting. Correlation between expected and actual reporting as an average was calculated and the staff received weekly updates on their observation-reporting results.

Results: The huddle board became a hub in providing an overview of the expectations of observations. To visualize the impact of the intervention, use of a run chart gave comprehensive information about the extent to which the expected goal of documentation was reached. Four different organizational steps and one individual action in the last step were taken to improve the observation-reporting. The identifying of the nonreporting nurses and individual staff guidance to these nurses resulted in a significant improvement in observation-reporting. The expected goal of $100 \%$ average reporting was achieved 6 months after all wards were included in the improvement project.

Conclusion: The combination of huddle boards, educating the entire staff in observation and documentation, and frequent feedback to the staff about the outcome proved to be a useful approach in medication safety work in nursing homes.

Keywords: patient safety, long-term care, prescribing, huddle board, nursing staff, systemapproach, medication-effect documentation

\section{Background}

Medication administration and management in nursing homes is a complex process where many different professionals are involved. ${ }^{1}$ Errors can occur during all phases of the medication process, which often is described as prescribing, dispensing, and administration of medicine. ${ }^{2}$ Rarely is the observation of the effect of the medication addressed. Nurses play a key role in medication safety. ${ }^{3}$ However, in nursing homes the nursing staff involved in the medication management hold a variety of educational levels. They are involved in the daily care of patients and are expected to observe the patients. Traditionally, the majority of the nursing staff in a long-term-care (LTC) facility has not been involved in systematic observation and reporting of medication effects
Correspondence: Stephan Ore Norlandia, Fjellstuveien 64, NO-0982 Oslo, Norway

Email Stephan.Ore@norlandia.com 
and side effects even if they feel that medication management should be a part of their job. ${ }^{1}$

Institute of Medicine (IOM) releases of the well-known report "To Err is Human"4 have become the point of departure for identifying patient safety issues and consequences of medication errors for patients as a main focus to address for the years to come. ${ }^{5}$ Improvements have been identified, however, the follow-up report 15 years later still points out that we have not reached as far as expected. ${ }^{6}$ Outcomes from safety work and systems for reporting of errors have mainly addressed a hospital focus. ${ }^{7}$ Less attention has been given to the primary health care and LTC. ${ }^{8}$ With the advent of an increasing elderly population living with chronic illness, and the policy initiatives that LTC should not take place in hospitals, ${ }^{9}$ it has become evident that safety issues must also be addressed in the long-term sector.

A UK report emphasizes that systems are not designed with safety in mind. The use of the huddle board as a communication tool in patient safety work has been introduced and proved to be effective for many safety issues. ${ }^{10}$ Huddle boards are used for a variety of purposes in health care, but have a renewal in patient safety work and might be a tool for working with improvements in two major areas: building learning systems and cultural issues (http://www.ihi.org/education/InPersonTraining/PatientSafetyExecutive/PatientSafetyExecutiveDevelopmentMarch2016/Documents/IHI\%20 Framework for Safety.pdf). A large number of health care providers are involved in working contexts characterized by highly inter-professional collaborators within and across levels of care. The safety framework opens up for addressing available communication tools for ensuring quality and continuity in patient care. ${ }^{11}$ It is highlighted that patient safety work addressing medication management needs to take a systems approach, compared to only having an individual approach where the individuals are blamed for the errors. ${ }^{12}$ Common for many studies addressing improvements for medication management in nursing homes, interventions for reducing polypharmacy, as for example ${ }^{13}$ or use of inappropriate drugs ${ }^{14}$ have been emphasized and developed. Use of multidisciplinary teams to perform systematic medication reviews has proved to identify and resolve drug-related problems in nursing homes. ${ }^{15,16}$ However, studies addressing targeted observation and reporting of medication effect in daily use for improving physicians' decisions in prescribing have not been identified. In a search in publications, from all institutional-health settings, methods to increase all nursing staffs' involvement were not identified. In the nursing home in this study, the registered nurses and physicians complained that they frequently had to make treatment decisions based on scanty observations, which made it harder to give efficient and safe treatment.

In Norway, where the current study was conducted, the authorities at the national level have initiated a patient safety program with several areas. One of the initiatives is the introduction of huddle boards for use in the clinical field. The huddle board is a tool aimed for keeping an overview of risks, status, and outcome of patient safety issues. We were not able to find publications that investigated the outcome of use of huddle boards in medication management in Norwegian nursing homes. Thus, in this project, we investigated the introduction and use of huddle boards for improving efficiency and safety of medication management in nursing homes by systematic feedback.

The aim was to investigate whether an introduction of a systematic use of huddle boards led to a higher amount of documentation in the electronic patient record (EPR) of observations regarding effects and side effects following a change in medication.

\section{Methods}

The project was conducted in a nursing home in Oslo, Norway, with a total of 151 LTC residents, divided into six wards (five somatic wards and one dementia ward). The average age of residents when the project started was 87 years, whereof $76 \%$ were women. The residents had poly-morbidity and were dependent on 24-hour care. The nursing staff involved in this project consisted of 28 who were equivalent to fulltime employment (FTE) registered nurses, 83 FTE auxiliary nurses, and 18 FTE nursing assistants. A project team consisting of the nursing home physician, the chief of quality, the team leader of the intervention wards, and one registered nurse was responsible for the project and designed the steps in the intervention. All nursing staff were included in the project, making no distinction in their level of formal training.

We collected baseline data on all the staff reporting on medication observations in the patient records from each ward during a period of 3 months. All six wards had the risk boards and risk huddles in use for more than 1 year, prior to the improvement project. The huddle board was introduced in the nursing homes' quality work and contained information about a variety of critical tasks and risk factors as well as what the patients themselves had stated to be of importance for them. Whether or not notification of important medication changes should be notified under the heading of "Medication change" was up to the nurse in charge or the physician to decide. The nurses and physician reflected on and prioritized 
when to ask for or when to stop this targeted observationreporting. It was decided that only very important and risky medication changes should be noted on the board. Usually 1-3 weeks of observation were being reflected upon at the weekly huddle-board meeting following the doctors' round.

The ward with the lowest reporting during this period was chosen to be the first ward exposed to the novel method, using the huddle board as a tool for identifying patients who needed to be observed for medication issues. The remaining five wards were used as controls: two were measured weekly as the intervention ward and the three remaining were measured only sporadically.

\section{The intervention}

Step 1: The huddle board was placed in the ward's main staff room. A systematic but brief list of effects/side effects of main groups of medicines was marked on the huddle board. The staff received short group training $(2 \times 20$ minutes for the intervention ward) in how they should use the list to improve their involvement in observational reporting. The training addressed how the nurse staff should use the information on the huddle board and how to produce short written reports in the EPR after each shift on the residents they had been responsible for during their shift. The huddle board was marked with three different magnet colors: yellow indicating that the patient was at risk. Information about the most critical symptom to observe (for instance "Pain") and where to report the observation in the EPR was provided. The staff had to check in the patient's EPR for further instructions. A red magnet meant "unresolved" indicating a need for special and continuing observation. A blue magnet was set up when the problem was resolved.

The risk boards were actively used daily during the handover between shifts. The responsible nurse had a "round" with the huddle board and pointed out critical tasks and risk areas on the risk board. Every staff member on every shift, whether unskilled or a fully trained registered nurse, had three to four residents as their primary responsibility. The responsible nurse instructed the next shift staff to memorize the information on the board for those residents they had the primary responsibility for on the coming shift. All the staff members were expected to document their observations in the patient record.

To measure if the intervention led to higher documentation of observations in the EPR regarding effects and side effects following a change in medication, we counted the weekly number of observations documented in the EPR, in percent of the requested number of observations. Typically,
3-7 residents out of a total of 25 were under this targeted observation on each ward. These patients were marked out on the huddle board with the yellow, red, and blue color system.

Step 2: Once a week during the first 4 weeks of the intervention period, the staff received an update on their observation-reporting results. This was done by presenting the run chart over the last week's reporting achievements. The results of the charting were discussed with the staff.

At this point, an unexpected event occurred which forced us to change our initial planned design in the project. As the word got out among the nonincluded ward leaders about the effect of the intervention, they all insisted on receiving weekly feedback to their own staff. This resulted in spread of the intervention earlier than we had planned. Thus, we were not able to keep the other wards as controls. The three next steps of the intervention were therefore introduced simultaneously on all the six wards.

Step 3: Introduction of an improved version of EPR for care planning provided a tool for the nurses to report electronically on all the wards. This new version was intended to ease the reporting of the medication observations.

Step 4: We introduced a specification of the frequency and time of observation of the individual patient. This was coded on the huddle board by a red-colored letter D (to make it clear the day shift needed to be observed and reported), A (for the afternoon shift), and $\mathrm{N}$ (night shift) as shown in Figure 1, leaving no doubt about the individual nurses' responsibility of observation-reporting on any shift. Expected documentations were counted weekly. If full observations by all shifts were notified on the board

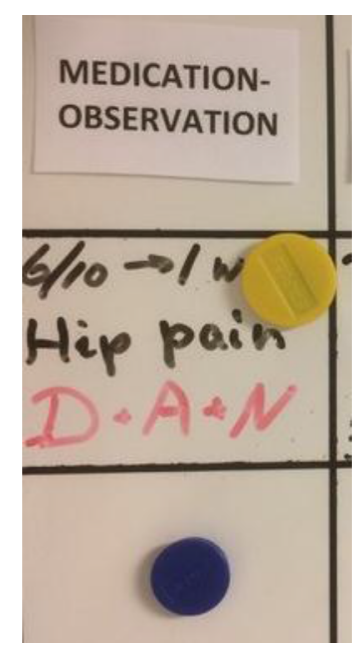

Figure I Illustration of a risk patient marked yellow and the expectations of reporting in the patient record. 
for $\mathrm{D}, \mathrm{A}$, and $\mathrm{N}$, from 1 week to the next, it was expected to find 21 ( 3 shifts $\times 7$ days $=21$ documentations) in the patient medication file. Once a week - on the day of the doctor's round - the nurse counted how many observations had been documented. As an illustration, if we counted 18 reports out of 21 expected, this was presented to the staff as $86 \%$ coverage. Every result above $80 \%$ was considered excellent: a result at this level was experienced to be sufficient for the nurse and physician to decide the effect of the medication.

Step 5: In this last intervention, the nonreporting staff members were identified and given individual colleaguebased guidance to enhance their reporting.

\section{Analysis}

Initially, we counted process indicators by absolute numbers of reports, but we experienced that this did not take into account the differences of observation needs from week to week and between the different wards. After introducing the coding $\mathrm{D}+\mathrm{A}+\mathrm{N}$ system 24 weeks after the intervention started, we were able to calculate the correlation between expected and actual reporting as an average. We found it relevant, if not very precise, to estimate in retrospect what reasonably would have been the goal prior to making it explicit. By doing this, we could follow a timeline from the beginning of the project, during the intervention period and afterward.

\section{Ethics}

Every health institution in Norway has, according to Norwegian legislation, the obligation to perform quality improvements. It is the institutions themselves which are responsible to approve quality projects and ensure they are within the ethical and confidentiality legislation. The head of the nursing home and the quality manager approved the reported project. All the data collected and used in this paper are presented in an aggregated form. No information could be connected to any individual patient or staff member.

\section{Results}

The huddle board became a hub in providing an overview of the expectations of observations. The first three steps described in the Methods section were introduced with only a monthly interval due to time limitations at the onset of the project. Following the run chart, with each ward separately, made it possible to visualize the impact of the different interventions.

In Figure 2, the intelligible run diagram - illustrated how it was presented from one ward - shows the percentages of expected and actual reporting of medication observations

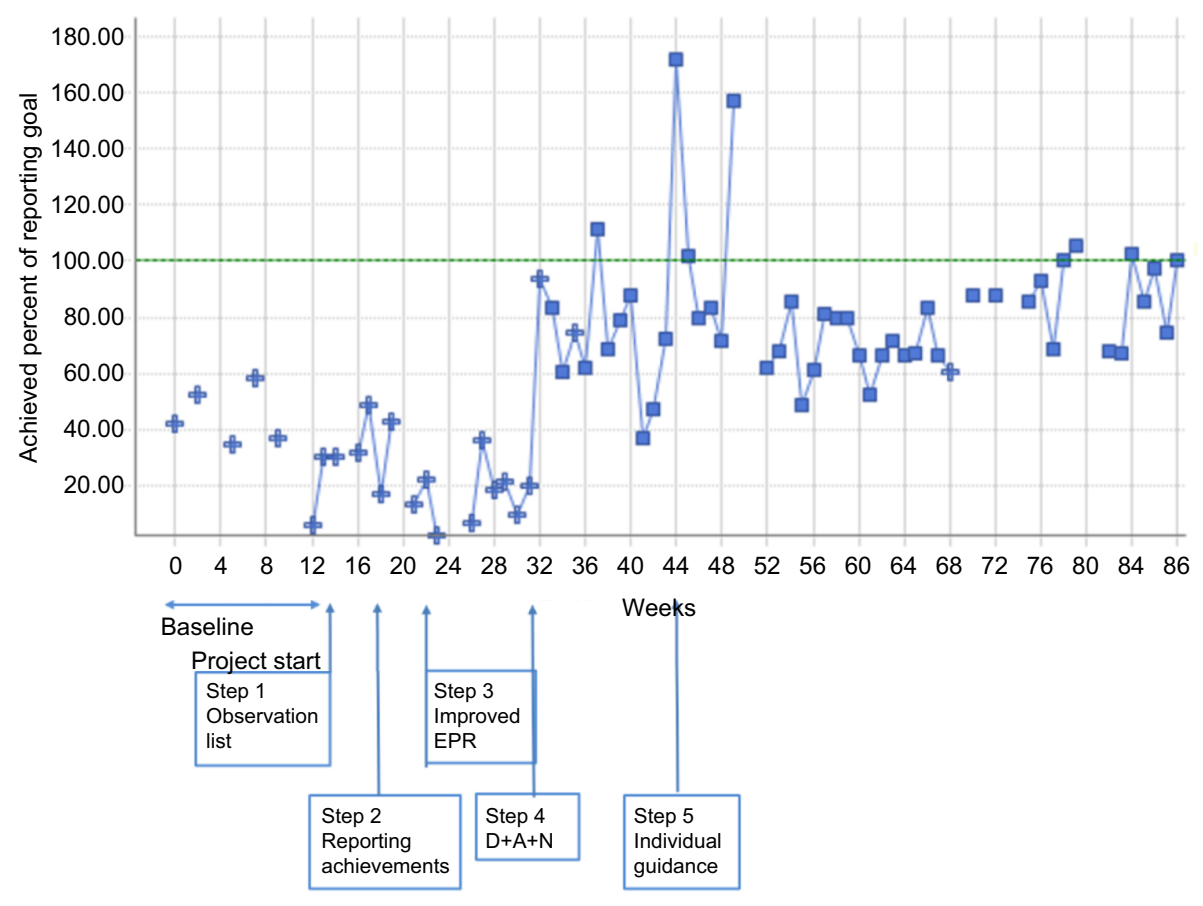

Figure 2 The percentage of reporting during the intervention period. Abbreviation: EPR, electronic patient record. 
and reporting during a period of 86 weeks. In the diagram, expected achievement was set to $100 \%$.

The diagram shows the different organizational steps (1-4) and individual actions (step 5) that were taken to improve the reporting. The diagram was sensitive to changes and gave comprehensive information about the extent to which the expected goal of documentation was reached. Unforeseen events were reflected in the extent of documentation. One event before the intervention was introduced was that the nursing home had to receive 20 fire evacuees from a neighboring nursing home. In this period, there was a decrease in the medication documentation (week 16). An opposite - and surprising - effect was during summer vacation when the documentation increased.

The period for implementing the intervention was conducted within a very short timeline; ie, 4-8 weeks. Ongoing motivation and pressure on the observation-reporting culture would also have a gradual effect and make it hard to discern the rapid interventions effects, when analyzing the run diagram. But, after extending the project period by 6 months, and then introducing the $\mathrm{D}+\mathrm{A}+\mathrm{N}$ which was explicitly clarifying the task, we did see a clearer shift in the run diagram.

An important finding was the impact of step 5 where the nonreporting nurses were identified and given individual guidance. After this effort, we observed a significant improvement in observation-reporting, and in fact met our goal of $100 \%$ average reporting only 6 months after all wards were included in the improvement project. In Figure 3, we show the impact of reporting on one ward where this approach was most significant.

\section{Discussion}

Our findings show that introducing and applying huddle boards combined with regular feedback to the staff about the outcome was useful for improving the documentation of medication observations in patients' records. Having in mind that medication management covers the whole process from prescribing to administration, ${ }^{2}$ the findings from our study only show to what extent observations have been documented. We have not explored the accuracy of the content of the medication documentation nor to what extent the prescribing routines has reduced errors on the individual level. This needs to be investigated in future projects. However, the lesson learned by combining three approaches; using the huddle board systematically, involving the entire staff, and providing frequent feedback to the staff, seems to have facilitated an improvement of the medication-effect documentation culture.

The use of huddle boards is shown to improve communication and outcome in safety work. ${ }^{10}$ The three-level tiered huddle theory of Goldenhar et al is developed for hospitals but is still useful as a comprehensive framework for understanding our safety work in a nursing home. The theory acknowledges that the medication management is a collaborative, inter-disciplinary, complex, and on-going effort where the macro-meso-micro levels need to be emphasized. Communication and collaboration between providers are required and in particular for older patients with complex health problems. ${ }^{17}$ We anticipated a positive effect by empowering and including the entire staff in the safety work and thus involving auxiliary nurses and nursing assistants in the project. Traditionally, they have not been involved in observing medication effects and side effects on patients. ${ }^{1}$ Hughes and Lapane reported that one of five - regardless of whether one is a nurse or nursing assistant - experienced that errors were a personal issue. Therefore, taking a systems approach, all observations from all staff members on effect and side effect of medication were seen as crucial for making appropriate decisions (meso-level). Reaching the overall goal

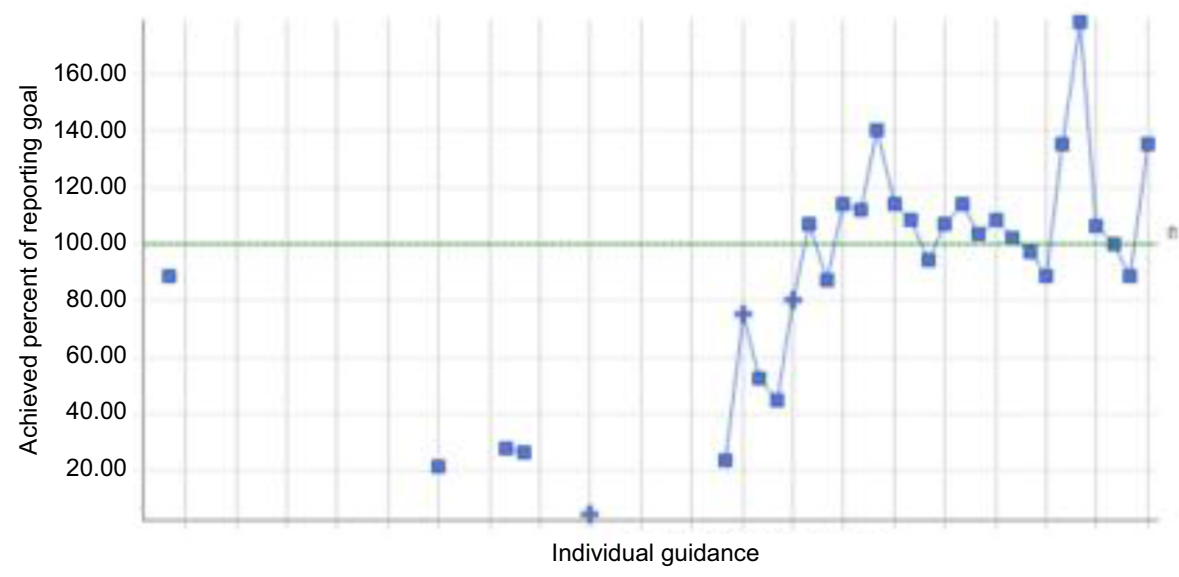

Figure 3 The influence on individual guidance in one of the wards. 
that all the staff members observed and documented as often as expected, may be a result of what Goldenhar et al state, that "huddle implementation can help systematize clinically related communication activities" (p. 904).

Huddle boards provide not only an easy access to shared information between all involved in the patient care but work also as a tool for the managers to have an easy overview on the extent to which the overall aim proposed at the nursing home (the macro-level) is put into practice. To prevent medication harm and improve practice, it is significant to have a valid and transparent measure and scalable mechanisms to collect and report outcome at all levels. ${ }^{6}$ In our project, the report system became a useful tool (Figure 2) for reporting the outcome of expected level of documentation. In addition, the responsible physician and head nurse at each unit (interaction between the micro- and macro-levels) used the information to identify those who did not follow-up documentation as expected. This resulted in tailored actions throughout the implementation process; ie, the head nurse approached those staff members who did not follow-up, until they had reached the standard. Taking individual action was a turning point for achieving our aim that all the staff should observe and report. We want to argue that the use of huddle boards links together staff members that hold a variety of skills and education level because it contributes to increased accountability and feeling of being connected to each other. ${ }^{10}$ Our project reveals that a connection between medication information documented in patient records at the individual patient level and the need for sufficient collective working processes is interdependent in medication management.

When we started the project, we were worried that the new observation-documentation routine could tire the nursing staff since it would add one more task to perform in an already loaded working day. ${ }^{18,19}$ Actually, we experienced that the project energized the staff. The fact that the nonincluded wards pushed the project team to include them shows that the intervention was highly valued and proved to become a method to scale up and spread. The enthusiasm we experienced on every ward when involving the staff in small-scale modification might provide ownership and thereby make adoption of a new method much more likely. In our project, we have not studied the staff's experiences, which should be conducted in greater depth, reaching a more comprehensive understanding. What we lost in standardization we won in involvement and implementation. In addition, the combination of stepwise implementation and tailored support for the individual nurses may have influenced the outcome resulting in $100 \%$ average reporting after 6 months. Our intervention seems to be sustainable because the medication reporting has stabilized around $80 \%-85 \%$ documentation on average, which provides information for making medication decisions. This level is nonetheless above the $20 \%-30 \%$ reporting activity in the baseline period.

A lesson to learn is the impact the intervention had on the lowest performing ward. This ward is still the lowest performer, but it also improved its performance significantly, and the spread to the other wards had its own momentum regardless of this. In hindsight, we think it is smarter to start with a ward most likely to adapt the new method, increasing the likelihood of having a success to facilitate spread to other sites.

We are convinced that the huddling has facilitated this change of the medication-effect documentation culture. Another lesson to be noticed is regarding the overall aim of quality work to improve resident safety or quality of life. We planned to use fall statistics as an outcome for our intervention, but falls seemed not to be influenced by our intervention. We experienced that falls varied independently to the staffs' observation performance. The falls occurred most likely due to an increasingly sicker and frail population with high risk of falling. For later safety work, it should be discussed carefully what adequate outcome within the available resources should be measured. To find changes of quality of life or patient safety indicators except for falls, would take time and unfortunately, personnel we did not have. We could not stretch our resources in a low-budget environment of a municipal LTC facility. We have continued to count performance almost every week after the project terminated because the staff expressed that it was useful. Thus, we have not seen staff coercion, actually more of a general positive involvement and it should be noticed that our sick-leave statistics have improved slightly.

The study has several limitations. We planned for an intervention with control groups to be able to generalize our findings, but the transparency within the nursing home made it impossible to follow the initial plan. However, we will argue that the nonintervention wards were aware of the results from the intervention, which anyway would have polluted our data collected from the control wards and been a threat to the validity of the project. On the other hand, despite the reality that this was not possible to conduct as recommended from a strict scientific point of view, ${ }^{20}$ we find the lessons learned to be important to share with others who plan for similar projects. It also shows how difficult it is to evaluate the effect of institution-based interventions for improving medication management. We cannot find that this problem is much accounted for in the national safety program although evaluation is pointed out as crucial in safety work. Therefore, 
our findings are important beyond our project and could be used to highlight the implication of how traditional safety work is designed and set out in practice. Since this was an improvement project and not regular research, we chose to take advantage of the enthusiasm among the other wards' staff and involve all six wards. Another limitation that should be noticed is that the first three steps of the intervention were introduced with only a monthly interval due to time limitations at the onset of the project. After all, we did have baseline data to compare the efforts to and make proper run diagrams for all six wards hereafter. This made us able to systematically measure and take actions during the evolvement of observation and documentation of patients' medication.

\section{Conclusion}

Increasing the observation and documentation of patient medication in nursing homes is crucial for providing a basis for reducing errors in prescribing medication. In our safety project we chose a three-layer approach; use of huddle boards, educating the entire staff in observation and documentation, and frequent feedback about the outcome to the staff. Our experience is that this method got the entire nursing staff engaged and involved in the medication observation-reporting in the nursing home. However, we need more information about the accuracy of the documentation and the effect on the patient level.

Registered nurses and physicians learn and can modify behavior through reading/studying. Auxiliary and assisting nurses may have a different approach to learning: more through experience and individual follow-up and guidance. The key to scaling up and spreading of proven work methods might be to give leeway to local modifications to enhance adoption and implementation.

\section{Acknowledgments}

We thank all the staff who participated in the project, the head nurses, and the coordinator for their valuable contribution. The project was conducted and supported by the Norwegian Authority Patient Safety Program for Educating Physicians in Safety Work.

\section{Disclosure}

The authors report no conflicts of interest in this work.

\section{References}

1. Dilles T, Elseviers MM, Van Rompaey B, Van Bortel LM, Stichele RR. Barriers for nurses to safe medication management in nursing homes. J Nurs Scholarsh. 2011;43(2):171-180.

2. Szczepura A, Wild D, Nelson S. Medication administration errors for older people in long-term residential care. BMC Geriatr. 2011;11:82.

3. Thomson MS, Gruneir A, Lee M, et al. Nursing time devoted to medication administration in long-term care: clinical, safety, and resource implications. J Am Geriatr Soc. 2009;57(2):266-272.

4. Institute of Medicine Committee on Quality of Health Care in America. Errors in health care: a leading cause of death and injury. In: Kohn LT, Corrigan JM, Donaldson MS, editors. To Err Is Human: Building a Safer Health System. Washington (DC): National Academies Press (US) Copyright 2000 by the National Academy of Sciences. All rights reserved; 2000.

5. Ulrich B, Kear T. Patient safety and patient safety culture: foundations of excellent health care delivery. Nephrol Nurs J. 2014;41(5):447-456.

6. Pronovost PJ, Cleeman JI, Wright D, Srinivasan A. Fifteen years after to err is human: a success story to learn from. BMJ Qual Saf. 2016;25(6):396-399.

7. Elkin PL, Johnson HC, Callahan MR, Classen DC. Improving patient safety reporting with the common formats: common data representation for patient safety organizations. J Biomed Inform. 2016;64:116-121.

8. Tsilimingras D, Rosen AK, Berlowitz DR. Patient safety in geriatrics: a call for action. J Gerontol A Biol Sci Med Sci. 2003;58(9):M813-M819.

9. European Commission. Long-term Care in the European Union. Brussels: EU; 2008.

10. Goldenhar LM, Brady PW, Sutcliffe KM, Muething SE. Huddling for high reliability and situation awareness. BMJ Qual Saf. 2013;22(11):899-906.

11. Dingley C, Daugherty K, Derieg MK, Persing R. Advances in patient safety improving patient safety through provider communication strategy enhancements. In: Henriksen K, Battles JB, Keyes MA, Grady ML, editors. Advances in Patient Safety: New Directions and Alternative Approaches (Vol. 3: Performance and Tools). Rockville (MD): Agency for Healthcare Research and Quality (US); 2008.

12. Wright $\mathrm{K}$. The role of nurses in medicine administration errors. Nurs Stand. 2013;27(44):35-40.

13. Olsson IN, Runnamo R, Engfeldt P. Drug treatment in the elderly: an intervention in primary care to enhance prescription quality and quality of life. Scand J Prim Health Care. 2012;30(1):3-9.

14. Hughes CM, Lapane KL. Nurses' and nursing assistants' perceptions of patient safety culture in nursing homes. Int J Qual Health Care. 2006;18(4):281-286.

15. Halvorsen KH, Ruths S, Granas AG, Viktil KK. Multidisciplinary intervention to identify and resolve drug-related problems in Norwegian nursing homes. Scand J Prim Health Care. 2010;28(2):82-88.

16. Davidsson M, Vibe OE, Ruths S, Blix HS. A multidisciplinary approach to improve drug therapy in nursing homes. J Multidiscip Healthc. 2011;4:9-13.

17. Steihaug S, Johannessen AK, Ådnanes M, Paulsen B, Mannion R. Challenges in achieving collaboration in clinical practice: the case of Norwegian health care. Int J Integr Care. 2016;16(3):3):3.

18. Storli M, Ingebrigtsen O, Nakrem S, Elstad TA. Safety measures for medication in nursing homes. Sykepleien Forskning. 2016;59801:e-59801.

19. Ellis W, Kaasalainen S, Baxter P, Ploeg J. Medication management for nurses working in long-term care. Can J Nurs Res. 2012;44(3):128-149.

20. Ringdal K. Enhet og mangfold. Samfunnsvitenskapelig forskning og kvantitativ metode. Bergen: Fagbokforlaget; 2001. 
The Journal of Multidisciplinary Healthcare is an international, peerreviewed open-access journal that aims to represent and publish research in healthcare areas delivered by practitioners of different disciplines. This includes studies and reviews conducted by multidisciplinary teams as well as research which evaluates the results or conduct of such teams or health care processes in general. The journal covers a very wide range of areas and welcomes submissions from practitioners at all levels, from all over the world. The manuscript management system is completely online and includes a very quick and fair peer-review system. Visit http://www.dovepress.com/ testimonials.php to read real quotes from published authors. 